\title{
Processes: Working Group Report
}

Stéphane Chatty, José Creissac Campos, María Paula González, Sophie Lepreux, Erik G. Nilsson, Victor M.R. Penichet, Mónica Santos, and Jan Van den Bergh

\section{Automatic Generation?}

It has often been suggested that model-driven development of user interfaces amounted to producing models of user interfaces then using automatic code generation to obtain the final result. However, this may be seen as an extreme interpretation of the model-driven approach. There are examples where that approach is successful, including mobile computing and database management systems. But in many cases automatic generation may be either impossible or may limit the quality of the final interface.

This debate raises the question of where information comes from in the design process:

- at one extreme is straightforward model translation or compilation: all the design information is contained in the model, and the automatic generation just produces the executable code.

- at the other extreme is a fully automated system that incorporates rules able to make the appropriate design choices: adapation to the task, the context and the available hardware.

- in between are more complex situations in which designers must bring additional knowledge and know-how. The process is thus a mix of human design and automated model translation.

Some authors suggest, following the second case above, that one can automatically translate a task model to a concrete user interface. However, task modelling languages currently are not capable of expressing all of the design information that is found in a concrete UI. For instance, some user interfaces rely heavily on the concept of sequence (wizards, for instance) when task modelling languages do not all have such a concept. Consequently, the information must either be encoded in a very rich set of rules, or added by a human designer. Therefore, until such very rich sets of rules are built, processes will most often consist of automatic translations interspersed with additions by human designers.

\section{$2 \quad$ Fitting UI into Larger Scale Processess}

The success of big industries relies on their processes, which have to be optimized for return on investment, efficiency and risk management. If one proposes to change existing processes by relying on user-centered methods, one will have 
to provide satisfactory answers to the following questions at least: what is the return on investment? How is the risk added by such methods managed?

- most decision makers are now convinced about the issue of return on investment, thanks to the success of the Web. The Web has been proved to them how better user interfaces yield more traffic, and they are ready to accept that they can improve their business too.

- as for risk, perception depends on the size of companies. Small companies are used to managing larger risks in their projects; therefore they are more open to accepting the potential risk brought by iterative design. Larger companies accept much smaller margins of risk; therefore, one needs to demonstrate that the proposed iterative processes improve the risk margin rather than degrade it.

Among the hurdles identified for integrating UI processes into larger development processes are:

- the fact that in classical software engineering, the architecture of the software is often designed before the analysis of user interface requirements, when there is a strong relationship between software architecture and the type of user interface that can be produced.

- the fact that the business logic is often developed before the user interface is designed, despite the fact that the implementation choices often impose severe constraints on user interaction

\section{Communicating with Users}

Although an abundant literature is dedicated to the role of users in design processes, there still appears to be a debate as to whether users are able to participate efficiently in the design process. Two opposed schools of thought emerge:

- some group participants consider that users bring a major risk to the design process because they keep changing their minds; these participants advocate processes that are user-centered but not with the actual participation of users;

- other participants contend that users are able to reason about abstract considerations when those have been properly introduced, are able to contribute efficiently to the design solutions when the appropriate prototyping and communication supports are used, and are no more difficult to manage than other actors of a development process.

These two schools of thought lead to very different views of the role of design managers. However, whatever the chosen design process it is important that this process is "self-evident" in that the customer or user knows what is the current phase of the process. In other domains (building architecture for instance), there is no ambiguity between the mock-up phase and the building phase. It should be the same in user interface design, using different levels of fidelity of prototypes. 


\section{Conclusions}

Based on the above discussions, the working group agreed that:

- there is no "silver bullet" process for developing user interfaces, especially not with full automation;

- HCI is about building new objects and not reproducing or evolving existing objects, which means that creativity will always play an important role;

- models should be used only when needed;

- models for communicating within the design and development groups and with users are essential;

- product design is a key activity in software development and its relationship to code development should be clarified. 